\section{Alfvén on heating by waves}

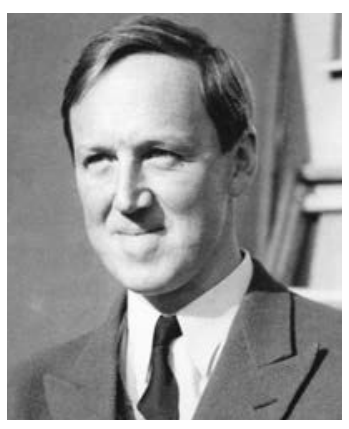

1 Ayoung Hannes Alfvén. (Wikimedia Commons)

\section{Ineke De Moortel, Isobel Falconer and Robert Stack explore the achievements and influence of Hannes Alfvén, in particular his seminal Monthly Notices paper of 1947 on the heating of the solar corona.}

bor he Swedish physicist Hannes Alfvén (figure 1) was born in 1908, into an era of polar exploration and the birth of nuclear physics. Frederick Cook's claim to have reached the north pole that year is disputed, but Robert Peary's success in 1909 was widely accepted. The Norwegian Roald Amundsen reached the south pole in 1911, and Robert Falcon Scott five weeks later in early 1912. Lord Kelvin, who had dominated Victorian physics, died in 1907; the concept of the electron, the first subatomic particle, was becoming established; and in 1908 the Geiger-Marsden experiment, in which alpha particles were back-scattered from gold foil, provided Ernest Rutherford with his first evidence of a nuclear atom.

Alfvén's family was intellectual, socialist and progressive (Pease \& Lindquist 1998); his parents were both doctors, one uncle was a composer, another an inventor and a third an agricultural scientist. He said that his interest in astronomy was aroused by Camille Flammarion's Astronomie Populaire, and in practical electronics through building receivers as a member of his school's radio club. In 1926, Alfvén entered the University of Uppsala, studying mathematics and physics. Here he was influenced by Manne Siegbahn, Nobel laureate in 1924 for his work on X-ray spectroscopy, and particularly by Carl Wilhelm Oseen, professor of theoretical physics, whose "Oseen equations" for flow of a viscous and incompressible fluid at small Reynolds numbers improve on Stokes's equations by including a correction term for inertial factors. The concern exhibited by Scandinavian physicists to model observed detail contrasts with the priority that Cambridge-trained mathematical physicists such as Kelvin, Stokes, and later Eddington and Chapman, assigned to mathematically beautiful theory; these differences of approach occur frequently in the careers of Alfvén and those whose traditions he inherited: Oseen, Birkeland and Størmer.

\section{Scandinavian tradition}

Alfvén obtained his doctorate in 1934 with a thesis on high-frequency oscillations in circuits containing a triode vacuum tube - an interest dating back to his adolescent experiences with radios. His continued work on electronic instrumentation for nuclear physics and cosmic-ray research up to the 1960s forms the backdrop for his more theoretical investigations (Pease \& Lindquist 1998). The years 1933-39 saw a stream of theoretical papers on the cosmic acceleration mechanism, first from Uppsala and later from the Nobel Institute for Experimental Physics in Stockholm, which Siegbahn founded in 1937. Alfvén

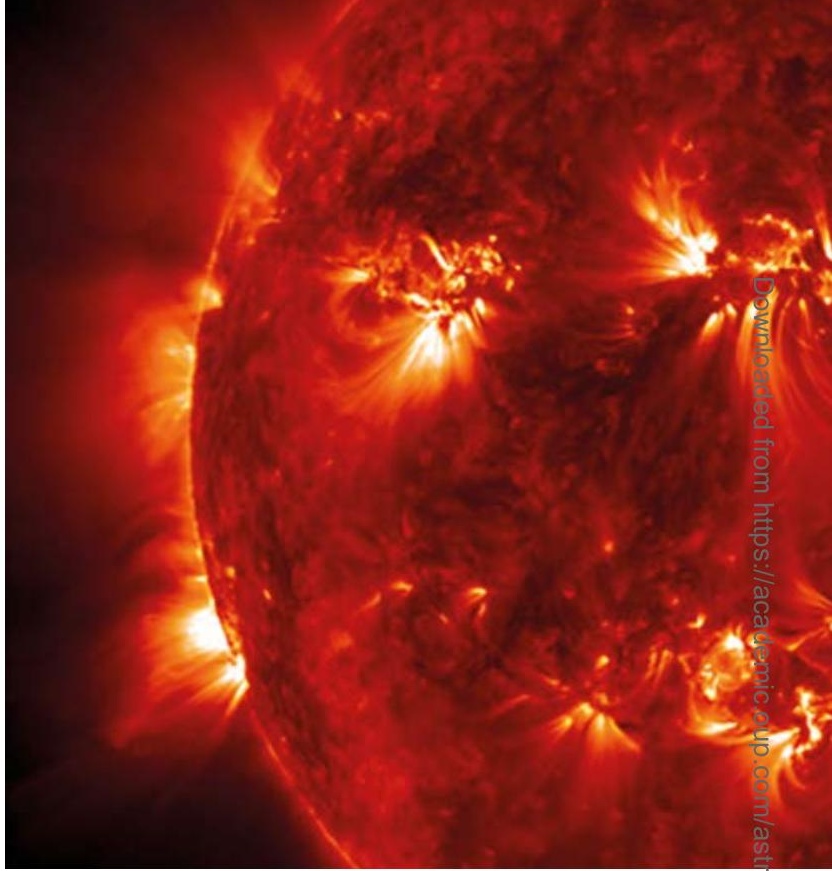

contended that cosmic rays originated in the local galaxy, the challenge being to suggest a plausible mechanism that would randomize their direction, ensuring the experimentally observed isotropic flux reaching the Earth. Although his proposal that an irregular galactic magnetic field, acting on electrically charged primary cosmic rays, might provide a suitable acceleration and randomization mechanism proved inconclusive, the existence of magnetic fields in interstellar space that confine most cosmic rays within the galaxy has been amply confirmed by observation.

Alfvén's interest in local space, particularly that between the Sun and the Earth, continued in 1937 with his first paper on aurora. Here he was heir to the ideas of the Norwegian Kristian Birkeland, who had given the first detailed and realistic account of the origin of aurora. It is hardly surprising that Alfvén, and Birkeland before him, had major interests in such phenomena. They lived at northern latitudes where aurora displays were frequent, visible and often spectacular. Polar expertise was widespread and widely recognized - the Swedish astronomer Anders Celsius was employed by the French expedition to Lapland as long ago as 1736; Amundsen and Fritjof Nansen were well known explorers of the early 20th century.

Birkeland himself had led two Arctic expeditions to gather experimental data and discovered a whole new class of disturbances, now known as magnetic substorms, that are little evident at the middling latitudes of most magnetic observatories (Egeland 1984). He suggested that these originated in local space as manifestations of field-aligned currents - vertically rising and descending currents of charged particles spiralling around magnetic field lines and connected by powerful horizontal currents that caused the actual storms. His ideas drew on his deep familiarity with electromagnetic theory.

To field observation and mathematical theory, Birkeland added laboratory experiment, completing a triangulation of approaches. His explanation of aurora originated in his 1896 experiments with cathode rays, which clearly demonstrated guidance of the rays by magnetic fields and were important to JJ Thomson the following year when he identified cathode rays as subatomic charged "corpuscles" (now known as electrons) (Birkeland 1896a, Thomson 1897). Birkeland (1896b) suggested that rays originating in the Sun were similarly guided by the Earth's magnetic field to the polar regions, causing the aurora and explaining the correlation between auroral activity and the sunspot cycle. He was not the first to make such a suggestion - Emil Goldstein, Henri Becquerel and the Danish meteorologist 


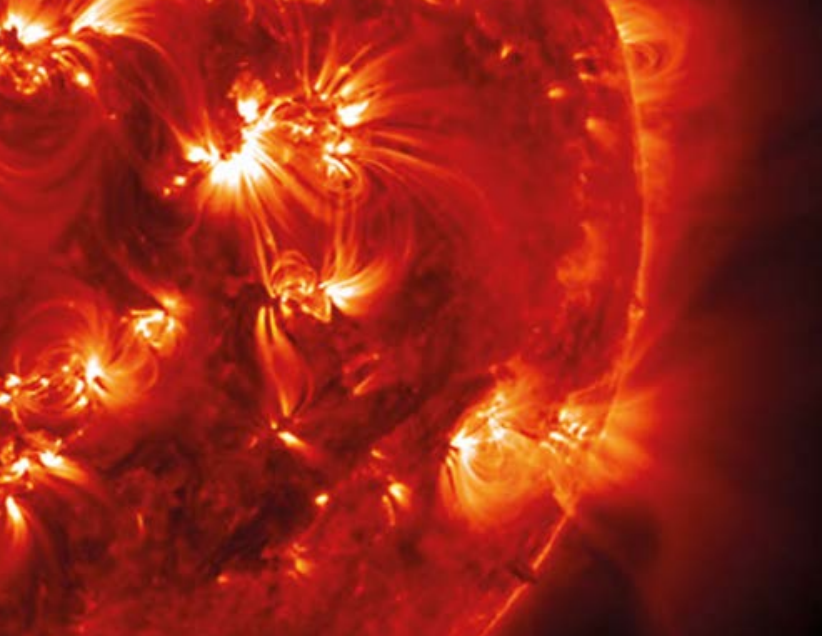

Adam Paulsen had all proposed similar ideas - but he was the first to back his ideas up with controlled experiments. Birkeland died in 1917, but he left behind the theory of field-aligned currents, together with a conviction that a marriage of observation, experiment and mathematics was the proper way to construct physical knowledge, which was to exert a powerful influence on Alfvén.

\section{Unfashionable science}

Entering the field in the late 1930s, Alfvén inevitably found the tide of scientific development against him for a variety of reasons: the field was unfashionable, the English-speaking world had achieved decisive dominance in physics through the inter-war period, and within the English-speaking community an established group of Cambridge-educated mathematical physicists were powerful opinion leaders.

With the benefit of hindsight, we can recognize a revolution going on in astronomy and cosmology in the middle of the 20th century. But at the time, the area was scarcely recognized as important, with only seven Nobel prizes throughout the entire century - and mathematical physics was completely overlooked (Karazija \& Momkauskaité 2004, Nobel Foundation 2020). Instead, the late 1930s to 1960 s was a golden age for nuclear physics, gaining 18 Nobel prizes overall - hardly surprising in view of its military prominence and funding during the second world war and subsequent cold war. This move towards nuclear physics is shown also in the co-citation networks analysed by Gingras (2007) as nuclear physicists Hans Bethe, Homi Bhabha and Gregory Breit succeeded quantum theorists of an earlier generation such as the Germans Werner Heisenberg, Arthur Sommerfeld and Friedrich Hund as the most central figures in physics 1937-44. The shift demonstrates also the dominance of the United States that was increasingly evident after the first world war. Martin (2015) identifies nuclear, high-energy and the beginnings of solid-state physics as the ascendant fields in the USA. The ascendancy was cemented by the immigration of many European scientists fleeing the Nazi regime - including the German nuclear physicists Bethe and Breit - and the stability and prosperity of the following years in comparison to war-ravaged Europe. English-language journals, particularly in the USA, grew rapidly at the expense of those in other languages in the 1930-45 period in both number of publications and frequency of citations (Gingras 2010).

Alfvén was far from the mainstream of physics in the 1940s. The difficulty in getting his voice heard was
2 The Sun in May 2015, imaged in extreme ultraviolet light $(171 \AA)$ by NASA's Solar Dynamics Observatory.

(Solar Dynamics Observatory/NASA)

exacerbated by the influence within the English-speaking space-physics community of Cambridge-trained theoreticians, especially Sydney Chapman (1888-1970). Chapman, after whom the Chapman Medal of the Royal Astronomical Society is named, entered geomagnetic research in 1917 (Taylor 1987, Chapman 1952). Birkeland's death that same year had left a vacuum that Chapman soon filled as the leading opinion-maker - which partially explains why Birkeland's ideas were neglected for the next 40 years even though he had been about to be nominated for a Nobel prize in 1917 (Egeland 1984, Potemra 1995).

Dessler (1984) suggests that Chapman's views were swayed by the legacy of Lord Kelvin, himself Cambridge-educated. Back in 1892, the elderly Kelvin had concluded, on energy considerations, that magnetic storms and sunspots must be unrelated phenomena, a conclusion opposite to Birkeland's proposal of four years later, as discussed above. Whatever the truth of Dessler's suggestion, Chapman adopted wholeheartedly the methods inculcated at Cambridge, devising problems that were well-posed mathematically, simplifying the physical situation to ensure rigorous solvability. His approach could be deemed a milder form of the "rationalistic cosmophysics" described by Kragh (2012). Kragh ascribes it mainly to Cambridge graduates - Chapman's student E Arthur Milne was a leading example - who believed in the power of pure reason based on a priori principles to construct cosmological knowledge. Opposition from more empirically oriented scientists erupted in Nature and spilled over into the RAS's review, The Observatory: "It is eventually borne in on the puzzled reader that Milne and [Arthur G] Walker are not trying to understand nature but rather are telling nature what she ought to be. If nature is recalcitrant and refuses to fall in with their pattern so much the worse for her" (McVittie 1940 p280).

Chapman did not go as far as Milne in disallowing observation completely, but his technique was to average large datasets to obtain subtle correlations such as the identification of lunar tidal effects in the ionosphere, in contrast to Birkeland's concern with spatial variation of individual events using approximation methods where necessary (Dessler 1984). Chapman's treatment of the effects of a plasma stream encountering the Earth's magnetic field, first as a plane perfectly conducting surface then as a cylinder, in order to enable exact mathematical analysis, displays sympathies similar to, though less extreme than, Milne's. Chapman restricted his model of the Earth's current system to a spherical shell about the Earth, allowing him to use spherical harmonic analysis, and to represent the geomagnetic data as Legendre polynomials. Dessler (1984) suggests that there must have been some "misinterpretation" of the experimental data to produce a good fit. It is not clear how much Chapman's decision owed to investment in particular mathematical methods and how much to a mistaken - or justificatory - claim that charged particles emanating from the Sun could not penetrate the conducting ionosphere. Either way, Birkeland's field-aligned currents just did not fit in to such an analysis. Nor, subsequently, did Alfvén's.

\section{Alfvén's contribution}

"With the benefit of

hindsight, we can

recognize a revolution

going on in astronomy

and cosmology in

the middle of the

20th century"
When Alfvén made his first notable contribution in 1939, proposing a theory of magnetic storms and auroras, Chapman was well established as a key gatekeeper. The paper was rejected by Terrestrial Magnetism and Atmospheric Electricity, the American Geophysical Union's flagship journal, because it disagreed with Chapman's theory; Alfvén had to publish in a Swedish journal instead (figure 3; Fälthammar \& Dessler 2006). Chapman had a reputation for quiet courtesy and for avoiding controversy (Cowling 


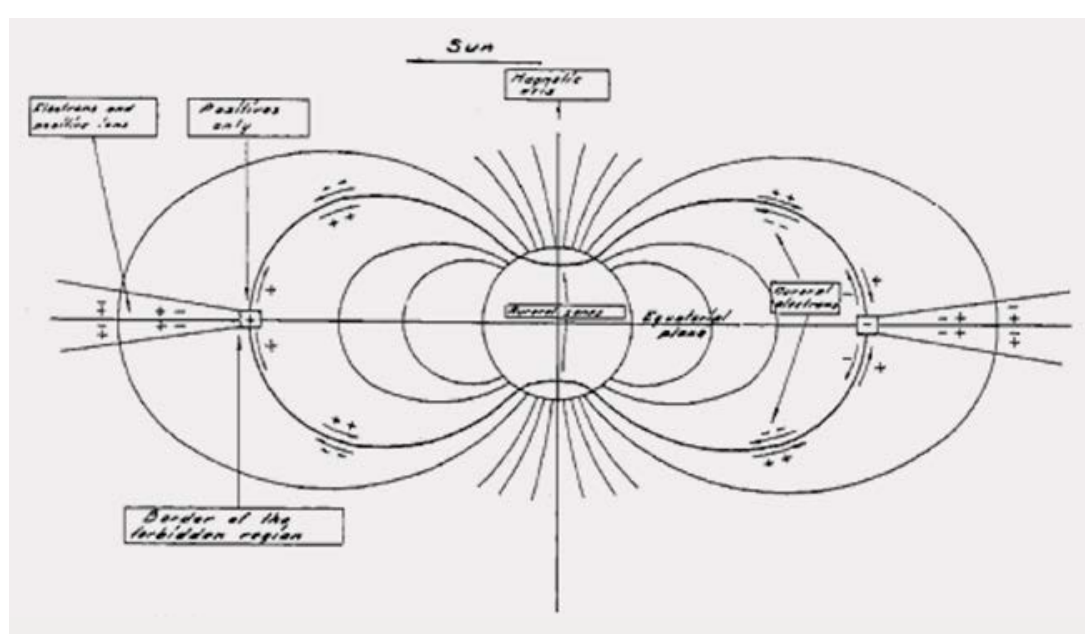

3 (Top) Alfvén's 1939 diagram - the discharge along the magnetic lines of force between the equatorial plane and the auroral zone. (Middle) A diagram of Chapman's mechanism. (Bottom) A diagram of Birkeland currents. (Alfvén 1939, Chapman 1927, Birkeland 1908)
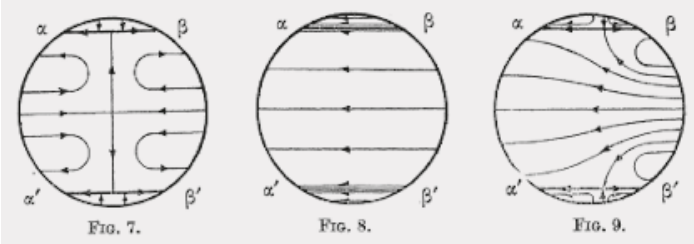
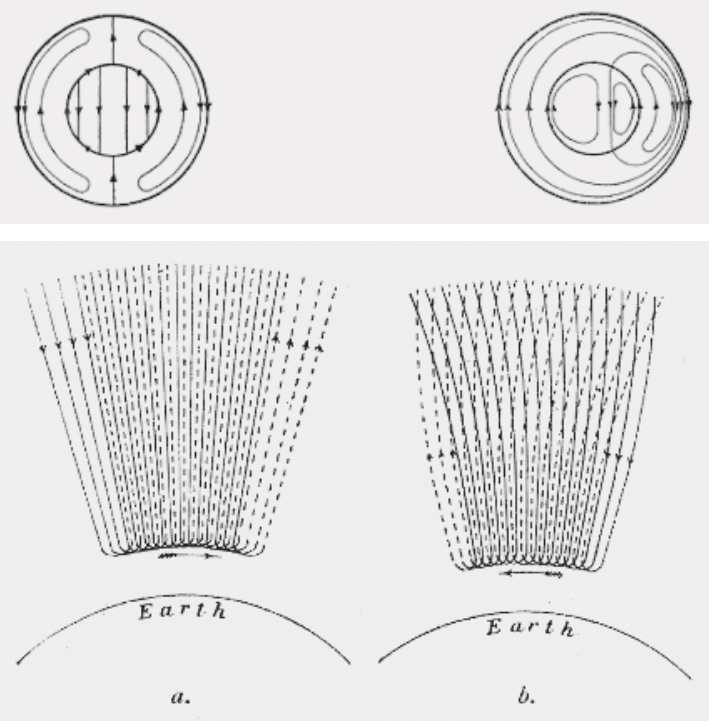

1971). But this very avoidance was immensely frustrating to the younger man, who felt it left no space for debate.

As late as his Nobel Lecture, Alfvén (1970) expressed views on theoreticians reminiscent of McVittie's attack on Milne quoted above: "Several of the basic concepts on which the theories are founded, are not applicable to the condition prevailing in cosmos. They are generally accepted by most theoreticians, they are developed with the most sophisticated mathematical methods and it is only the plasma itself which does not understand, how beautiful the theories are and absolutely refuses to obey them."

Three years later, in 1942, investigating the origin and motion of sunspots, Alfvén combined the equations of hydrodynamics with Maxwell's equations for electromagnetism to model the motion of a highly conducting gas in a magnetic field. He established that the magnetohydrodynamics (MHD) equations implied the existence of electromagnetic-hydrodynamic waves, which we now know as "Alfvén waves": transverse, incompressible perturbations with magnetic tension as the restoring force, which travel along the magnetic field at the Alfvén speed $\left(v_{A}=B / \sqrt{\mu \rho}\right)$ (Alfvén 1942). An excellent contemporary review of this work can be found in Russell (2018).

Almost at the same time, Grotrian (1939) and Edlen (1942) established that the highly ionized iron lines observed during a total solar eclipse must imply coronal temperatures in excess of a million Kelvin. Thus was born the "coronal heating problem": with the temperature at the solar surface only of the order of $6000 \mathrm{~K}$, how do temperatures in the atmosphere of the Sun (and many other stars) reach several million Kelvin? The question provided an immediate domain of applicability for Alfvén waves.

In his seminal 1947 paper in Monthly Notices on "Granulation, magneto-hydrodynamic waves, and the heating of the solar corona", Hannes Alfvén put forward the idea that the photospheric granulation "must produce magnetohydrodynamic [Alfvén] waves, which are transmitted upwards to the chromosphere and the corona". Although Alfvén here estimates that the wave energy is only of the order of $1 \%$ of the energy radiated by the Sun, he nevertheless suggests that "it is possible that the very high temperature found in the corona is produced through this magnetohydrodynamic [wave] heating".

Alfvén was not the only one to consider heating by waves as a possible explanation for the million-degree corona. For example, Biermann (1946) and Schwarzschild (1948) proposed that the transport and subsequent dissipation of energy by acoustic waves ("noise") produced by the convection zone (just beneath the solar surface) could be a potential heating mechanism for the solar atmosphere. What sets Alfvén's paper apart is the idea that perturbations of the Sun's magnetic field provide the energy source for coronal heating, at a time when the link between bright active regions in the solar corona and strong magnetic fields was not yet established.

If the above theories feel obvious or familiar to readers, it is good to remember that the concept of heating by waves was put forward among other, more exotic theories, such as heating by meteors falling into the Sun's atmosphere "from interstellar space". It was envisaged that these meteors would acquire such high speeds that "when stopped", they could produce the observed coronal temperatures. However, as Alfvén remarks in his 1947 article: "It is very dubious, however, if the number of meteors suffice to cover the thermal losses of the corona." More importantly though, Alfvén states that "the shape of the corona depends upon the solar activity, which makes it likely that the corona is produced by the Sun itself". In other words, Alfvén understood that the source of energy for the extremely high temperatures in the solar atmosphere must lie within the Sun itself, independent of external agents.

\section{Observing Alfvén waves}

The existence of Alfvén waves was not immediately widely accepted, in part for reasons discussed above. However, during a visit to Chicago in 1948 (giving a seminar about cosmic rays), Alfvén had the opportunity to discuss the concept of Alfvén waves with Fermi, who readily accepted their existence (Fälthammar \& Dessler 2006). The combination of Fermi's standing in the scientific community as a Nobel laureate and the publication of his paper on cosmic radiation (Fermi 1949) led to international interest and widespread acceptance of Alfvén waves.

Two further elements are particularly relevant for the early phase of Alfvén's hypothesis of an Alfvén wave-based heating mechanism for the solar atmosphere. In 1958, Parker inferred the existence of the solar wind, suggesting that the solar corona is not in static equilibrium but is constantly expanding outwards. As soon as the existence of the solar wind was confirmed by early space-based observations in the first half of the 1960s, observations also showed that the solar wind is full of Alfvén waves. This reinforced Alfvén waves as a credible candidate for coronal heating, as well as for the acceleration of the solar wind.

Secondly, in the same timeframe, Cowling (1953) and Piddington (1956) demonstrated that the form of 
(a) $\mathrm{Ca}$ II H
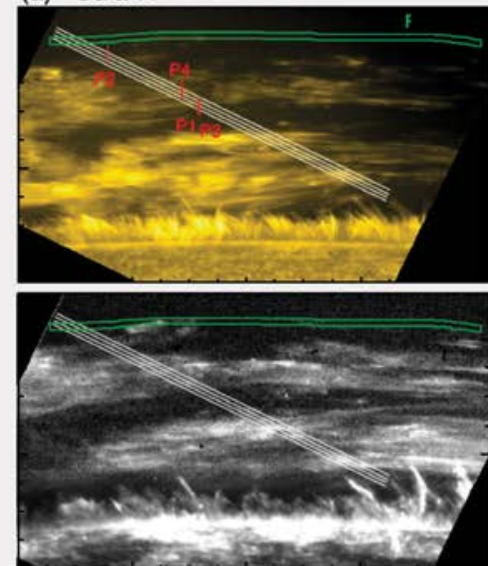

(b) Si IV (c) $\mathrm{Mg}$ II $\mathrm{k}$
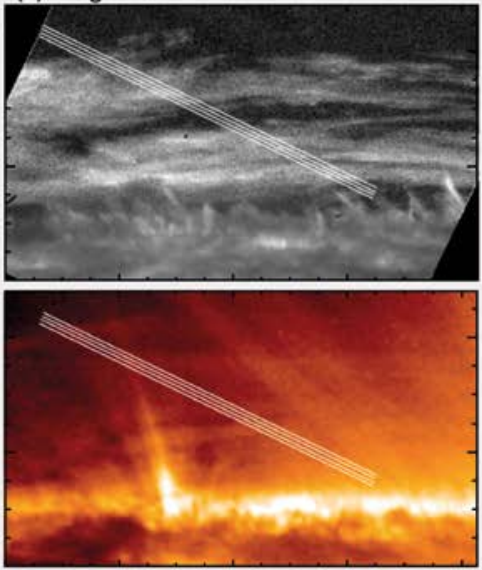

(d) $\mathrm{Fe} \mathrm{IX}$

\section{(C) Motion of prominence plasma crossing the slit}
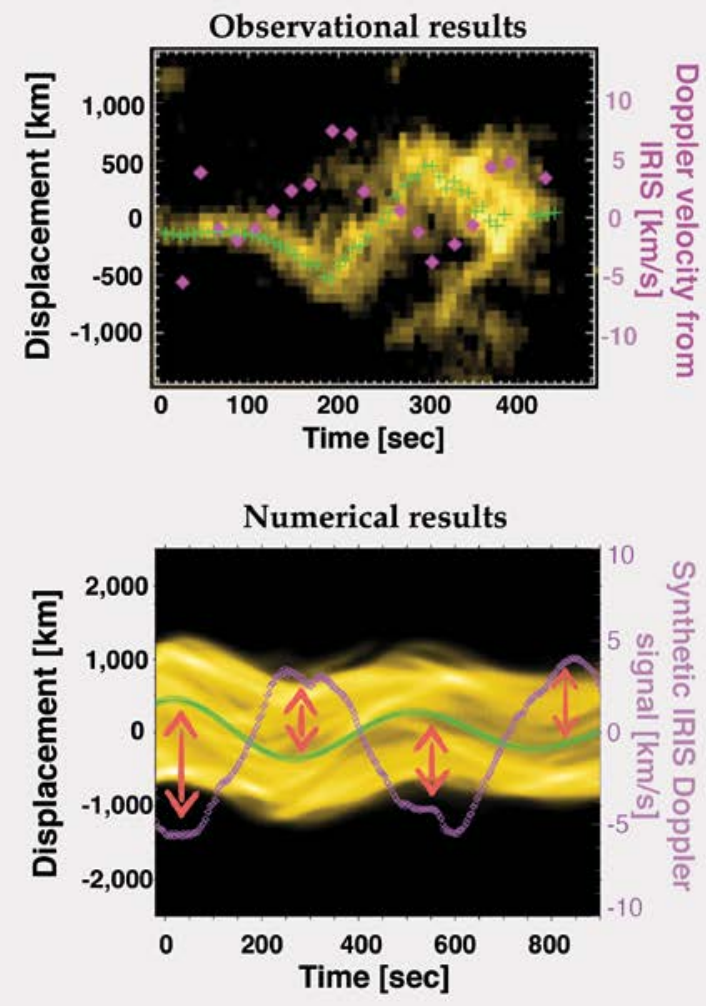
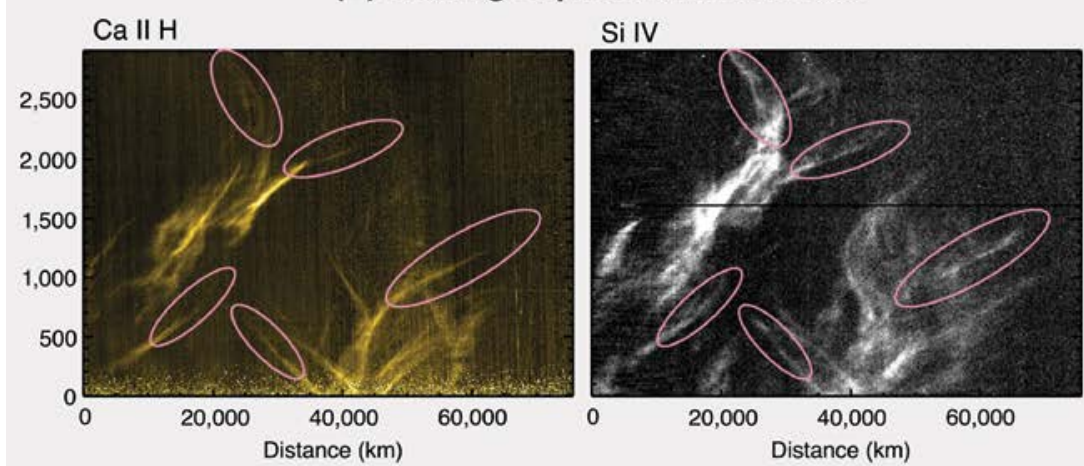

4 Prominence observed on the southeast solar limb on 19 October 2013. (A) (a) A Hinode/SOT image of the observed prominence in Ca II H ( 10000 K). The horizontal elongated structures are prominence threads. The four white lines are the positions of the IRIS slit. The green box indicates the region used for tracking the horizontally moving threads for panel B. The vertical red bar P1 is the selected location for the space-time plots in panel C. (b) The prominence as observed in IRIS Si IV ( 80000 K). (c) The IRIS Mg II image (10000-15000 K). (d) The SDO/AIA image in the Fe IX passband image ( 1 MK).

(B) (Left) A space-time plot of the Hinode/SOT Ca II observation along the green box shown in the panels in A. The intensity in the green box is summed vertically to include the entire part of oscillating threads. (Right) The same as the left panel, but for the IRIS Si IV data. Several locations (indicated by circles) show the transition of temperature from cool to hot, where the threads in the Ca II data are visible first and as they fade away, co-spatial threads appear in the Si IV data. (C) Space-time plots of Hinode/SOT Ca images at the red bar marked P1 in panel A. The green crosses are the central positions of the threads. The purple diamonds indicate the LOS velocity derived from Mg II k spectra at the corresponding positions of the thread centre. Clear oscillations are visible with a $180^{\circ}$ phase difference between the transverse motions and the LOS velocities. The bottom panel shows the corresponding model result, obtained by forward modelling the computational simulations. (Figures modified from Okamoto et al. 2015 and Antolin et al. 2015)

resistivity used by Alfvén in his 1947 paper overestimated the amount of dissipation and hence the heating that would result from Alfvén waves in the solar atmosphere. In fact, Piddington (1956) states that both resistive (Joule) and viscous heating are negligible in the solar atmosphere. In perhaps a somewhat contradictory fashion, Alfvén waves retained their status as a credible, potential mechanism to heat the solar corona. Instead, attention shifted to addressing the slow dissipation rate, a search that to some extent is still going on today (see e.g. Arregui 2015). MHD wave research in the 1980s and 1990s was dominated by the development of wave models with an enhanced dissipation rate, mostly based on the ideas of resonant absorption (e.g. Ionson 1978) and phase-mixing (Heyvaerts \& Priest 1983) that facilitate the creation of small length scales and hence accelerate the wave dissipation, which occurs most efficiently at small length scales.

Trying to summarize the current status of Alfvén wavebased heating of the solar atmosphere is not an easy task. So, let us think about the key ingredients that are needed. The first one would be identifying (observing) Alfvén waves in the solar atmosphere. Next, we need to assess the energy budget contained within these waves and, finally, to address the slow dissipation rates.
From the mid-1990s onwards, the spatial and temporal resolution of space-based missions such as $\mathrm{SoHO}$ (the Solar and Heliospheric Observatory, launched on 2 December 1995, only eight months after Alfvén's death) and TRACE (Transition Region and Coronal Explorer) resulted in observations of resolved wave motions in the solar atmosphere. Most of the oscillations observed initially, though, were either associated with (sporadic) impulsive events and/or contained very little energy and hence did not seem particularly relevant in the context of coronal heating. The field of coronal seismology (or magnetoseismology) however, put forward as early as Uchida (1970) and Roberts et al. (1984), flourished. Combining wave models and observations, seismology provides insight into the local plasma conditions, including the magnetic field strength, in the solar atmosphere (see e.g. Nakariakov \& Verwichte 2005 or De Moortel \& Nakariakov 2012).

This changed from 2007 onwards when several papers revealed the presence of "Alfvén waves" in solar X-ray jets (Cirtain et al. 2007), the chromosphere (De Pontieu et al. 2007), prominences (Okamoto et al. 2007) and large coronal loops (Tomczyk et al. 2007) in both ground- and space-based observations. Although the interpretation of the observed oscillations (and many more that followed) 
5 Figure 2 from Alfvén's 1947 paper depicting the energy change with distance from the centre of the sun. (RAS/OUP)

\section{"Without in situ data, the interpretation of waves and oscillations relies heavily on combining observations and modelling"}

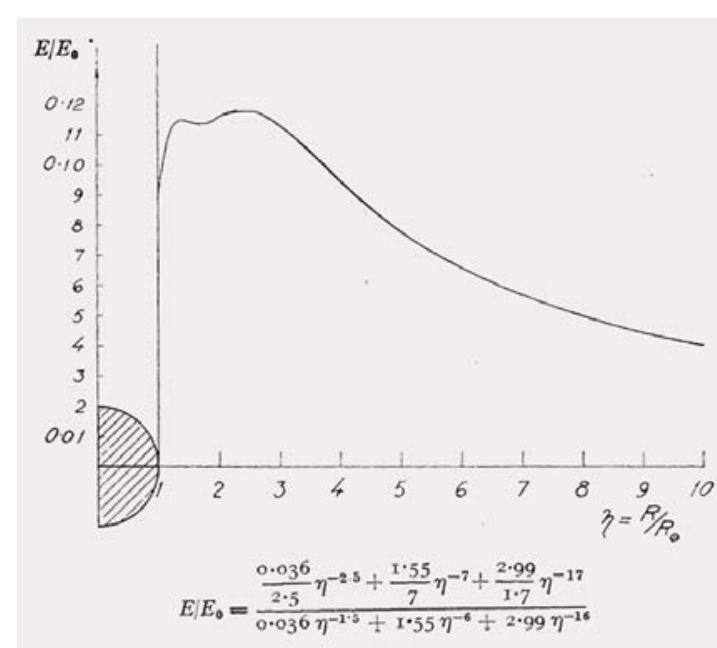

as Alfvén(ic) waves was not without controversy (see box "Identifying Alfvén waves"), these observations sparked a renewed interest in Alfvén waves as a potential heating mechanism; many of the observed waves appear to contain a substantial amount of energy and, unlike the impulsively excited oscillations described above, are present for long periods of time.

Without access to in situ measurements, the interpretation of observed waves and oscillations relies heavily on combining observations and modelling. An elegant example of this approach can be found in the papers by Okamoto et al. (2015) and Antolin et al. (2015); observations from IRIS (Interface Region Imaging Spectrograph) and Hinode/SOT (Solar Optical Telescope) of a prominence showed an anti-phase relationship between the line-ofsight (Doppler) velocity and the transverse motions in the plane-of-the-sky and this relationship was reproduced by forward-modelling a computational model of Alfvénic waves that combined resonant absorption, phase-mixing and the Kelvin-Helmholtz instability (see figure 4).

In another recent example, Grant et al. (2018) interpret observations by the Interferometric BIdimensional Spectrometer (IBIS) at the Dunn Solar Telescope as evidence of heating of chromospheric plasma in a sunspot umbra by Alfvén waves through the formation of shock fronts. The authors suggested that the dissipation of mode-converted Alfvén waves driven by upwardly propagating magnetoacoustic oscillations is consistent with observed local temperature enhancements of $5 \%$.

Both of these examples relate to observations at chromospheric temperatures. In the hotter corona, analysis of CoMP (Coronal Multi-channel Polarimeter) observations by Morton and collaborators (2016) has recently demonstrated the presence of enhanced power around $3 \mathrm{mHz}$, regardless of the local topology, which the authors interpret as evidence of a link between the observed coronal transverse perturbations and the global solar p-modes. Using CoMP observations over an extended period of time (2012-15), Morton et al. (2019) recently reported on the possible presence of a basal Alfvénic flux throughout the solar cycle.

The examples above are selective and intended to provide only a flavour of the current state of the art; while remote sensing makes mode identification a difficult task, observations have now established the ubiquitous nature of these Alfvénic waves and oscillations in the solar atmosphere.

\section{Energy budget}

Crucial to assessing the role of Alfvén waves in heating the solar atmosphere is an accurate assessment of the energy contained in observed waves and oscillations. Again, this is not trivial to derive from remote-sensing observations and estimates associated with observed Alfvénic waves reported so far differ substantially. For example, CoMP observations of propagating disturbances in off-limb coronal loops suggest an energy budget that is several orders of magnitude too small to account for coronal heating (Tomczyk et al. 2007). In contrast, analysing SDO/AIA observations, McIntosh et al. (2011) report that the observed small-amplitude oscillatory displacements contain sufficient energy to account for the heating requirements of the quiet Sun and coronal holes $\left(-1-2 \times 10^{5} \mathrm{erg} \mathrm{cm}^{-2} \mathrm{~s}^{-1}\right)$. However, in active region loops the energy budget is estimated to be at least an order of magnitude too small $\left(10^{5} \mathrm{erg} \mathrm{cm}^{-2} \mathrm{~s}^{-1}\right.$ vs $2 \times 10^{6} \mathrm{erg} \mathrm{cm}^{-2} \mathrm{~s}^{-1}$ needed to account for the heating of coronal loops, Withbroe \& Noyes 1977).

A possible reason for the discrepancies in the reported energy budgets could be the effect of superposition of Doppler velocities, both along the line of sight and within lower-resolution instruments, which would result in a significant proportion of the wave energy flux being "hidden" in the large (observed) non-thermal line widths (see McIntosh \& De Pontieu 2012). This effect was demonstrated using 3D numerical simulations of oscillations in cylindrical fluxtubes by De Moortel \& Pascoe (2012), who found that the superposition of randomly directed oscillating transverse displacements could lead to discrepancies of around an order of magnitude or more in the estimated line-of-sight energy budget. In addition, mode identification further complicates deriving an estimated energy budget from observed waves and oscillations (Goossens et al. 2013).

The presence of even substantial amounts of wave energy does not necessarily imply that these waves can contribute efficiently to the local heating (see e.g. Arregui 2015). The difficulties currently being tackled by modelling efforts could be grouped broadly into four categories (Cargill et al. 2016):

- Damping vs dissipation In a complex and dynamic environment such as the solar atmosphere, processes such as mode coupling and resonant absorption can lead to the transfer of energy from one wave mode to another. This might be observed as rapid damping of, for example, an observable transverse oscillation, by transferring the energy of this displacement into (hard to observe) azimuthal perturbations in the boundaries of the loop. However, this damping does not necessarily imply dissipation (and hence heating) on the same rapid timescale.

- Dissipation The key obstacle here is to rapidly transfer the wave energy from the (observed) large-scale wave motions to sufficiently small length scales where dissipation is effective (for accepted values of the Reynolds number). Recent efforts have focused on creating such a cascade of energy in models that, in increasing degrees, take into account the complexity of the real solar atmosphere. For example, Alfvénic perturbations reflecting off internal (longitudinal) density structuring can lead to a turbulent-like pattern of counter-propagating waves. Similarly, the generation of the Kelvin-Helmholtz instability at the edges of transversely oscillating loops can create complex vortex structures. In both examples, fine-scale currents become widely distributed throughout the loops. - Self-consistency Typically, wave models investigate perturbations to an assumed equilibrium. Mechanisms such as phase-mixing and resonant absorption require the presence of a gradient in the Alfvén speed, which models usually assume to be caused by a gradient in the local (transverse) density - i.e. the loop is denser than the environment. Heating through these mechanisms is most efficient at the locations of the steepest gradients, which in such a setup are at the edges of the loop. Radiation, on the other hand, is stronger in higher-density regions, i.e. the core of the loop. Therefore, unless heating can somehow 


\section{Identifying Alfvén waves}

The detection of Alfvén waves in the outer solar atmosphere is not trivial. Without access to in situ measurements (i.e. the ability to directly relate measurements of velocity and magnetic field perturbations), the interpretation of observed waves and oscillations as Alfvénic is based upon the transverse and incompressible nature of the observed perturbations, combined with the fact that they are propagating along the magnetic field at speeds of the order of the local Alfvén speed. However, in a "flux tube" (an idealized representation of a coronal loop) standard MHD wave theory would interpret such waves as kink waves, rather than the corresponding Alfvén waves in a uniform medium. On the other hand, one could attribute the "Alfvénic" character of such waves to the dominance of magnetic tension as the restoring force, in which case kink waves in a low-beta flux tube and Alfvén waves in a homogeneous medium can both be considered as special cases of Alfvénic waves.

In addition, when transverse density structuring is present, the transverse Alfvénic kink wave mode couples to the azimuthal $(m=1)$ Alfvén wave in the shell regions of the loop. See, for example, De Moortel et al. 2016 for a review.

be distributed efficiently throughout the loop, the assumed equilibrium loop structure will not be self-consistently maintained by such a wave-based heating mechanism. - Feedback and efficiency Following local (wave-based) heating, thermal conduction along the field lines will rapidly transport heat to the lower (cooler) layers of the atmosphere, where it causes material to evaporate and hence alter the local density. This feedback process could potentially help distribute the localized heating through the loop rather than just at the edges of the loop, as a series of changes in the local density gradient leads to "drifting" of the heating layer (see e.g. Ofman et al. 1998). However, Cargill et al. (2016) demonstrated that this structuring occurred on timescales longer than the thermal evolution of the loop, i.e. processes such as cooling and draining are more significant than the wave-heating feedback. In addition, the authors found that transport coefficients need to be enhanced significantly to obtain effective heating in the first place.

\section{Still not clear}

More than 70 years after Alfvén first proposed the idea, the exact role Alfvén waves play in heating the solar atmosphere remains unclear. Although directly associating the damping of observed wave energy with dissipation and heating remains elusive, observations have shown without a doubt that Alfvén(ic) waves are present throughout

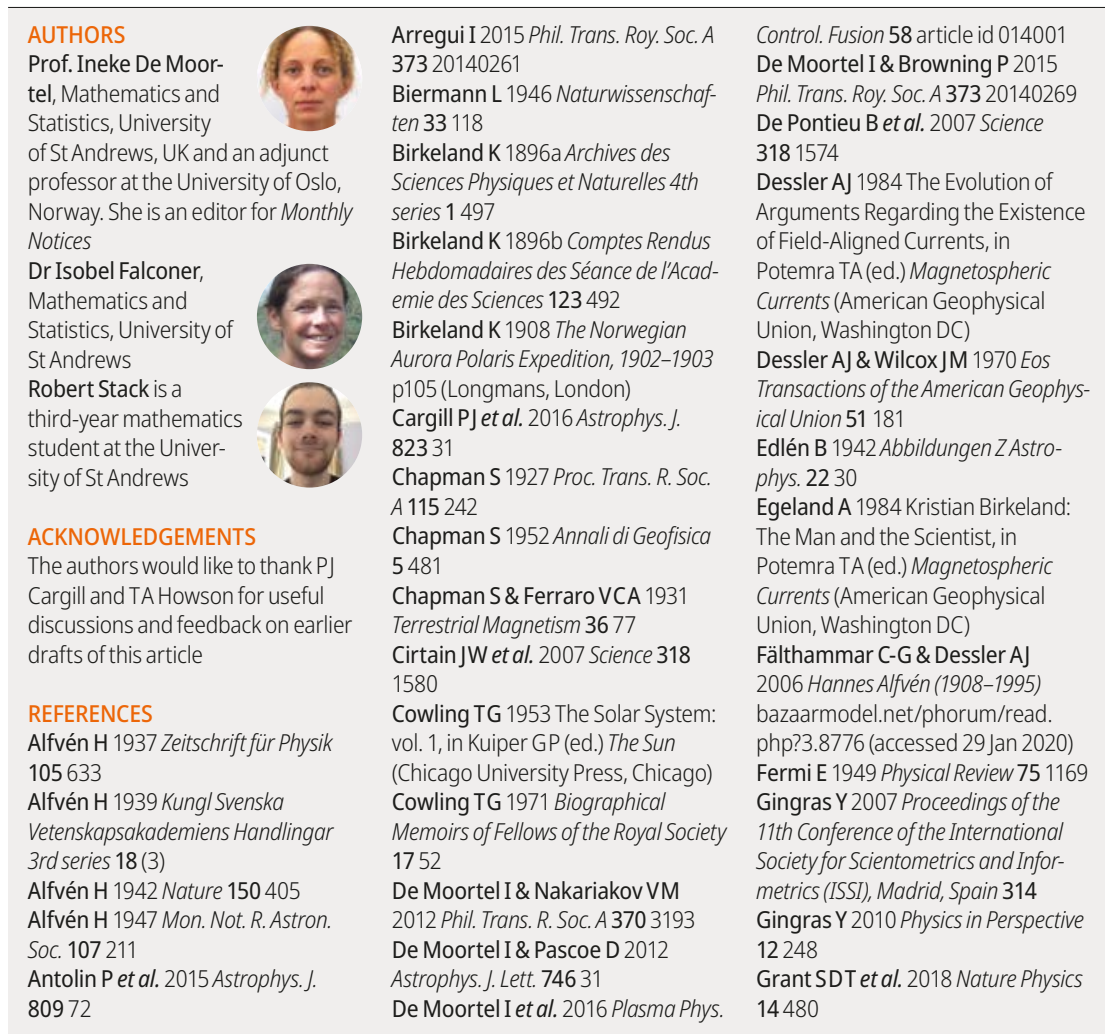

the solar atmosphere. It is more than likely that these waves and oscillations contribute to the heating of the solar atmosphere and the acceleration of the solar wind. How this contribution relates to other potential heating mechanisms is still not clear and, more than half a decade after Cowling and Piddington raised the issue, the slow dissipation rate of Alfvén waves remains a stubborn stumbling block. It is worth pointing out here that Alfvén did not propose Alfvén waves as the only coronal heating mechanism. Indeed, in his 1947 paper he writes "no doubt currents could be produced also in other ways", and "all these discharges give probably an essential additive heating of the chromosphere and especially the corona". It does not seem that Alfvén was proposing a solar atmosphere heated by any one mechanism exclusively. At the time of writing this article, the first observational results from Parker Solar Probe are available and the launch of Solar Orbiter is imminent. Both spacecraft will provide in situ measurements closer to the Sun than ever before. As the solar-physics community begins to grapple with these new observations and measurements, it seems likely that the painstaking task of unravelling the complex coronal heating problem will happen through the close interplay between multiwavelength observations and 3D computational simulations, underpinned by physical and mathematical models (see e.g. Klimchuk 2006, 2015, Parnell \& De Moortel 2012, De Moortel \& Browning 2015).

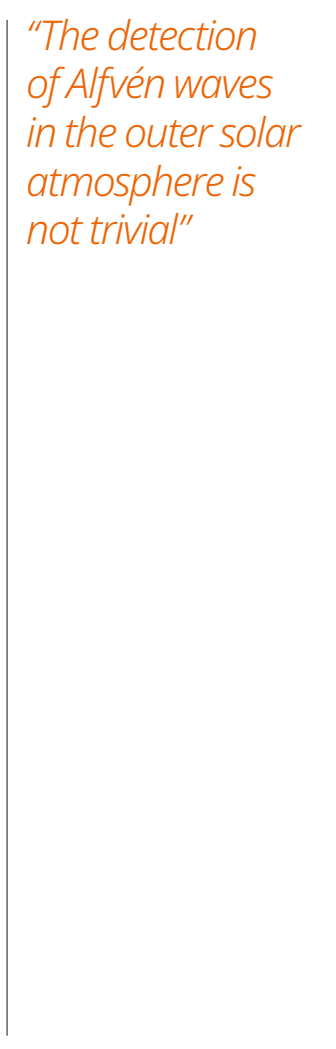

Grotrian W 1939 Naturwissenschaften 27214

Heyvaerts J \& Priest ER 1983 Astron Astrophys. 117220 Ionson JA 1978 Astrophys. J. 226650 Karazija R \& Momkauskaité A 2004 Scientometrics 61191 KlimchukJA 2006 Solar Phys. 23441 KlimchukJA 2015 Phil. Trans. Roy. Soc. $A 37320140256$ Kragh H 2012 Visions of Revolutions: Microphysics and Cosmophysics in the 1930s, in Epple M \& Müller F (eds) Science as Cultural Practice: Mod ernism in the Sciences ca. 1900-1940 (Oldenbourg Verlag, Munich) McIntosh SW et al. 2011 Nature 475477

McIntosh SW \& De Pontieu B 2012 Astrophys.J. 761138 McVittie G 1940 The Observatory 63273

Morton RJ et al. 2016 Astrophys. J 82889

Morton RJ et al. 2019 Nature Astronomy 3223

Nakariakov VM \& Verwichte E 2005 Living Rev. Solar Physics 23 Martin JD 2015 Nuclear High Energy and Solid State Physics, in Montgomery GM \& Largent MAA (eds) Companion to the History of American Science (Wiley, Chichester) Nobel Foundation 2020 All Nobel prizes in physics nobelprize.org/ prizes/lists/all-nobel-prizes-in-physics (accessed 26 Jan 2020) Ofman Let al. 1998 Astrophys. J. 493474

Okamoto TJ et al. 2007 Science 318 1577 Okamoto TJ et al. 2015 Astrophys. J. 80971

Parker EN 1958 Astrophys. J. 128664 Parnell CE \& De Moortel I 2012 Phil. Trans. Roy. Soc. A 3703217 Pease RS \& Lindquist S 1998 Biographical Memoirs of Fellows of the Royal Society 443

PiddingtonJH 1956 Mon. Not. R. Astron. Soc. 116314 Potemra TA 1995 Phys. Scr. 60107 Roberts B et al. 1984 Astrophys.J. 279857

Russell AJB 2018 Solar Phys. 29383 Schwarzschild M 1948 Astrophys. J. 1071

Taylor RJ 1987 History of the Royal Astronomical Society: Volume 2 1920-1980 (Blackwell, Oxford) Thomson JJ 1897 The Electrician 39104

Tomczyk Set al. 2007 Science 317 1192

Uchida Y 1970 Publ. Astron. Soc. Japan 22341

Withbroe GL \& Noyes RW 1977 Ann. Rev. Astron. Astrophys. 15363 